\title{
Two Novel Cascade $\Sigma \Delta$ Modulators for Broadband Low-Voltage A/D Conversion
}

\author{
Alonso Morgado, Rocío del Río and José M. de la Rosa \\ Instituto de Microelectrónica de Sevilla, IMSE-CNM (CSIC/Universidad de Sevilla), \\ Edif. CNM-CICA, Av. Reina Mercedes s/n, 41012 Sevilla, SPAIN. \\ Phone: +34955056666, Fax: +34955056686, E-mail: \{alonso|rocio|jrosa\}@imse.cnm.es
}

\begin{abstract}
This paper presents two new architectures of cascade $\Sigma \Delta$ modulators that, based on the use of resonation, increase their effective resolutions compared to previously reported topologies while presenting high robustness to non-linearities of the amplifiers and very relaxed output swing requirements. In addition, the use of loop filters based on Forward-Euler integrators, instead of Backward-Euler integrators as proposed in earlier approaches, simplifies the switched-capacitor implementation and makes the proposed architectures very suited for highly-linear broadband $\mathbf{A} / \mathbf{D}$ conversion $^{\dagger 1}$.
\end{abstract}

\section{INTRODUCTION}

The increasing demand for high data-rate $\mathrm{A} / \mathrm{D}$ converters for the next generation of telecom systems implemented in nanometer CMOS technologies is motivating the exploration of new topologies of wideband $\Sigma \Delta$ Modulators $(\Sigma \Delta \mathrm{Ms})$ [1]-[6]. Among others, the use of resonation [4] and/or unity $\underline{\text { Signal }}$ Transfer Function (STF) [2][3] are demonstrating to be good candidates for low-voltage implementation. On the one hand, loop-filter resonators allow to increase the effective resolution as compared with integrator-based noise-shaping filtering. On the other hand, by making STF unity, the integrators ideally process quantization error only, thus relaxing their requirements of amplifier gain non-linearity and output swing.

The above-mentioned strategies can be combined with cascade topologies in order to increase the order of the modulator whereas keeping stability and operating with low $\underline{\text { OverSampling }}$ Ratio $(O S R)$ [5][6]. However, the implementation of in-loop resonators requires using Backward-Euler (BE) or non-delayed

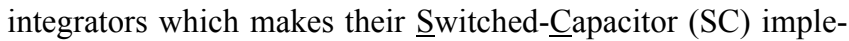
mentation more difficult.

This paper presents two novel topologies of cascade $\Sigma \Delta \mathrm{Ms}$ intended for high-speed and low-voltage applications. Both modulators combine resonation techniques and unity STF. One of them is based on local resonation whereas the other one employs global resonation. In both cases, Forward-Euler (FE) instead of BE integrators are used, thus circumventing the implementation problems presented in the former architectures based on cascaded resonators.

The paper is organized as follows. Section II provides a background on previously reported resonation-based cascade $\Sigma \Delta \mathrm{M}$ architectures, which make use of unity STF and resona-

$\dagger 1$. This work has been supported by the Spanish Ministry of Science and Education (with support from the European Regional Development Fund) under contract TEC2007-67247-C02-01/MIC, the Spanish Ministry of Industry, Tourism and commerce (FIT-330100-2006-134 SPIRIT) and the Andalusian Ministry of Innovation, Science and Enterprise under contract TIC-2532. tion. Section III describes the $\Sigma \Delta$ topologies proposed in this paper. Finally, behavioral simulations are shown in Section IV that demonstrate the benefits of the proposed architectures in terms of effective resolution and robustness to amplifier non-linearities and relaxed output swing requirements.

\section{RESONATION-BASED CASCADE $\Sigma \Delta \mathrm{M}$ TOPOLOGIES}

An efficient way to increase the resolution without penalizing the number of integrators consists of including resonators inside the modulator loop filter. Thus, the so-called local resonation technique has been used in $\Sigma \Delta \mathrm{Ms}$ considering either single-loop [4] or cascade topologies [5]. In both cases, this technique allows to shift the zeroes of the Noise Transfer Function (NTF) from DC, thus distributing them in an optimum way such that the in-band noise can be minimized [1].

In the case of cascade architectures, only the last stage normally uses resonation in order to reduce the complexity of the digital cancellation logic. As an illustration, Fig. 1 shows a cascade with local resonation in the last stage. This topology, originally presented in [5], takes advantage of both the unity STF of the first stage and the feedforward path at the last one, thus obtaining relaxed output swing requirements.

Recently, a new kind of resonation strategy, named global resonation, has been applied to cascade $\Sigma \Delta \mathrm{Ms}$ [6]. This new approach, illustrated in Fig. 2 for a fourth-order cascade architecture, is obtained by feeding back the quantization error from the last stage to the previous one. Note that this topology achieves resonation thanks to a global path that feeds back a scaled version of the last stage quantization error at the input of the first stage quantizer.

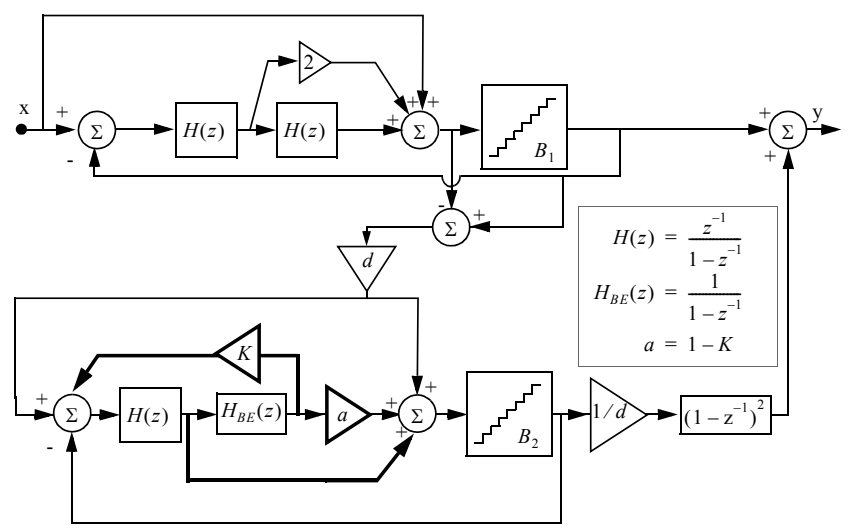

Figure 1. Fourth-order cascade $\Sigma \Delta \mathrm{M}$ architecture with local resonation and unity STF [5]. 
If a linear model is considered for the embedded quantizers, the $\operatorname{NTF}(z)$ of the modulators in Fig. 1 and Fig. 2 is given by:

$$
\operatorname{NTF}(z)=\frac{-\left(1-z^{-1}\right)^{2} \cdot\left[1-(2-K) \cdot z^{-1}+z^{-2}\right]}{d}
$$

where $d$ stands for the inter-stage gain. Note that the zeroes of $N T F(z)$ are a function of $K$, whose value can be optimally cho-

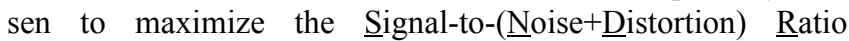
$(S N D R)$. Indeed, practical cases may lead to a resolution increase of up to approximately $10 \mathrm{~dB}[6]$.

Another conclusion that is derived from (1) is that increasing $d$ (to reduce the quantization noise) yields a reduction of the feedback coefficient $K / d$ (see Fig. 2). In practice, this results in a smaller capacitor ratio, which makes the electrical implementation more difficult and prone to circuit non-idealities.

\section{PROPOSED CASCADE $\Sigma \Delta \mathrm{M}$ ARCHITECTURES}

Previously reported resonation-based $\Sigma \Delta \mathrm{Ms}$ like those shown in Fig. 1 and Fig. 2 require using BE or non-delayed integrators, $\left[H_{B E}(z)=1 /\left(1-z^{-1}\right)\right]$, thus complicating the electrical implementation. Instead, the proposed architectures use both local and global resonation strategies and include loop filters based on FE integrators that are more suited for implementing $\mathrm{SC} \mathrm{A} / \mathrm{D}$ converters than previous reported topologies.

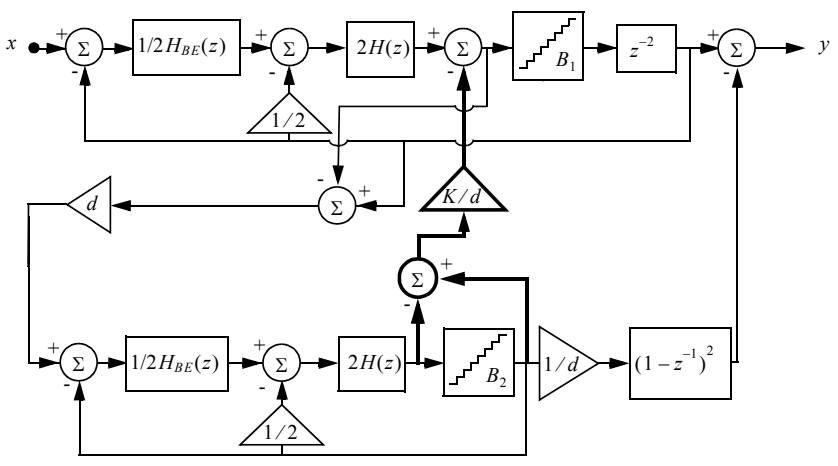

Figure 2. Fourth-order cascade $\Sigma \Delta \mathrm{M}$ with global resonation [6].

\section{A. Proposed cascade $\Sigma \Delta M$ with local resonation}

Fig. 3 shows the proposed (I) fourth-order $\Sigma \Delta \mathrm{M}$ architecture. This topology is a cascade architecture that implements local resonation with only FE or delayed integrators. The price to pay is that additional analog coefficients and a feedback path are needed. Note that this modulator is based on the one depicted in Fig. 1 with two modifications. The first one is the use of a second-order single-loop FE-integrator based resonator topology, proposed in [4], in the last stage of the cascade. On the other hand, the use of unity STF at the first stage allows feeding the second stage with only one branch [3][7].

\section{B. Proposed cascade $\Sigma \Delta M$ with global resonation}

Fig. 4 shows the second proposed topology, that makes use of unity STF at every stage whereas resonates through a feedback path from the last stage to the previous one, i.e. using global resonation.

However, as a consequence of using FE integrators, a scaled addition of the delayed last-stage quantization error is required at the input of the 1st-stage quantizer. Nevertheless, in order to overcome the implementation of the extra analog delay in Fig. 4 -and an additional inter-stage Digital-to-Analog Converter (DAC), marked with $\otimes$ for clarity, that provides the last-stage quantization error to the first stage - , a more efficient mode to

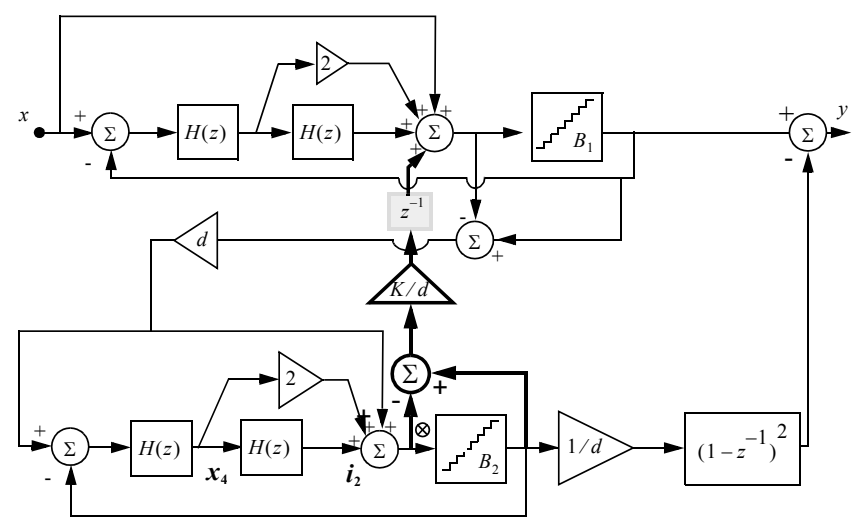

Figure 4. A first approach to the proposed (II) cascade with global resonation topology.

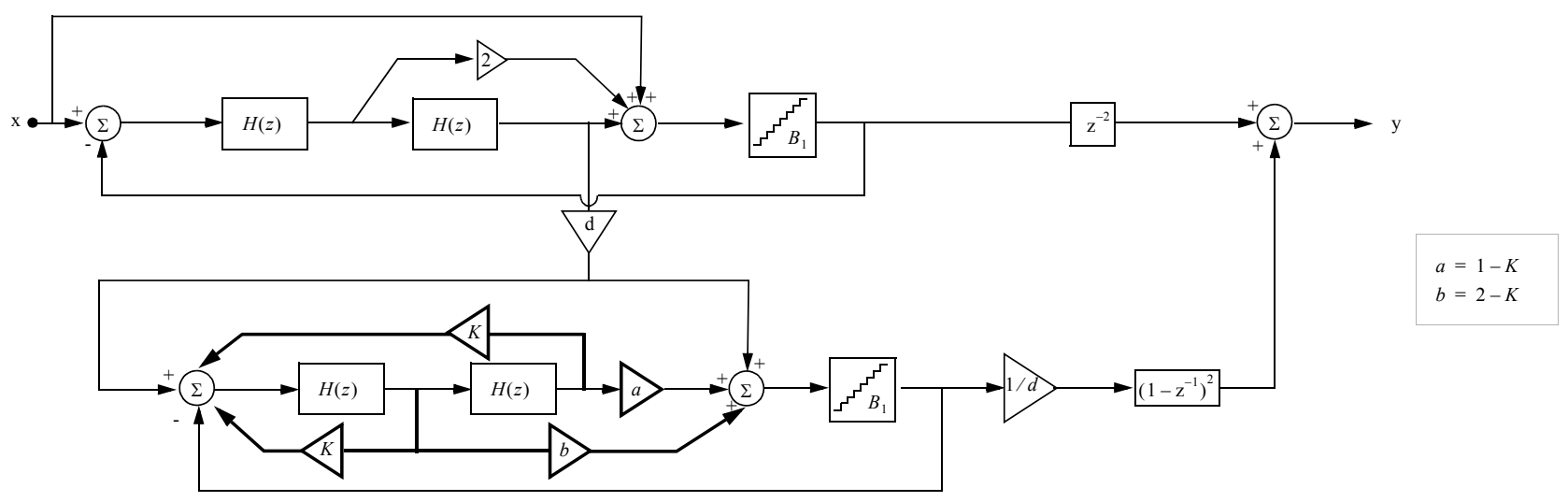

Figure 3. Proposed (I) cascade $\Sigma \Delta \mathrm{M}$ with local resonator. 
realize the global resonation with unity STFs stages is described below. Indeed, analysing the linearized Z-transform of Fig. 4, the delayed quantization error of the last stage can be directly obtained as

$$
X_{4}(z)+I_{2}(z)=-z^{-1} \cdot E_{2}(z)
$$

This modification results in the architecture depicted in Fig. 5. Note that, in addition to avoiding the use of both an extra analog delay and an inter-stage DAC, the $\Sigma \Delta \mathrm{M}$ in Fig. 5 requires only one analog coefficient $(K)$ to make the resonation, instead of the three coefficients $[K,(1-K)$ and $(2-K)]$ in Fig. 3, which simplifies the electrical implementation and reduces the sensitivity to circuit non-idealities.

\section{SIMULATION RESULTS}

In order to compare the performance of the proposed $\Sigma \Delta \mathrm{M}$ architectures (Fig. 3 and Fig. 5), several behavioral simulations has been done using SIMSIDES, a Simulink-based time-domain simulator for $\Sigma \Delta$ modulators [8]. All topologies operate with 4-bit internal quantizers, an inter-stage gain $(d)$ of 1 , a $1-\mathrm{V}$ reference voltage and a very reduced oversampling ratio of 4 . In addition, all the following simulations include $k T / C$ noise sampled by $0.25-\mathrm{pF}$ capacitors at the first integrator. Also, the embedded DACs are assumed to be linear.

As an illustration, Fig. 6 compares the effect of resonation on the output spectrum of the proposed topologies to that of a traditional 2-2 cascade (MASH), for a $-6 \mathrm{~dB}$ Full $\underline{\text { Scale }}$ (dBFS) input level. Note that, thanks to the resonation, a notch - clearly visible in Fig. $6 a$ - is produced close to the bandwidth in the proposed topologies.

The rounded optimal feedback coefficient that causes the resonation in the topologies depicted in Fig. 3 and Fig. 5 is $K=0.4$, resulting in a shift of two zeroes of the $N T F$ from 0 to $0.8 \pm j \cdot 0.6$. Because of this optimum distribution of the $N T F$ zeroes, the in-band noise is minimized. This is better illustrated in Fig. 7, where the SNDR is represented versus the input amplitude. In this example the use of resonation increases the resolution of the proposed architectures by roughly $10.5 \mathrm{~dB}$ within the whole input range compared to a traditional cascade.

Another advantage of the proposed architectures comes from the use of unity STFs, yielding to the subsequent reduction of the amplifiers' output swing. This is illustrated in Fig. 8 by plotting the histograms of the integrator outputs in the architectures proposed in this work and those corresponding to Fig. 2. In addition, a classical cascade 2-2 architecture is also included in the comparison for the sake of completeness. Note that the output swing requirements of the proposed topologies - only $0.15 \mathrm{~V}$ for the 1 st and 3rd amplifiers; and $0.1 \mathrm{~V}$ for the $2 \mathrm{nd}$ and 4th ones - are smaller than the ones for the modulator of Fig. 2 and for the traditional cascade. This is translated into a better linearity of the proposed architectures.
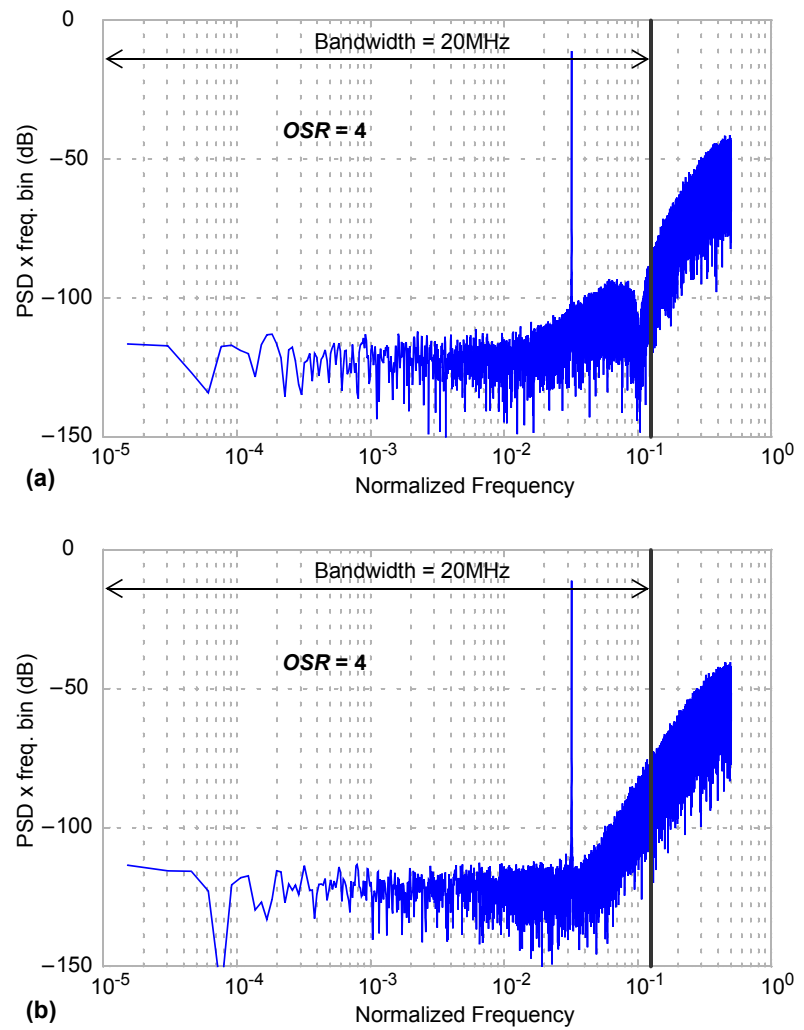

Figure 6. Modulator spectrum: (a) with and (b) without resonation.

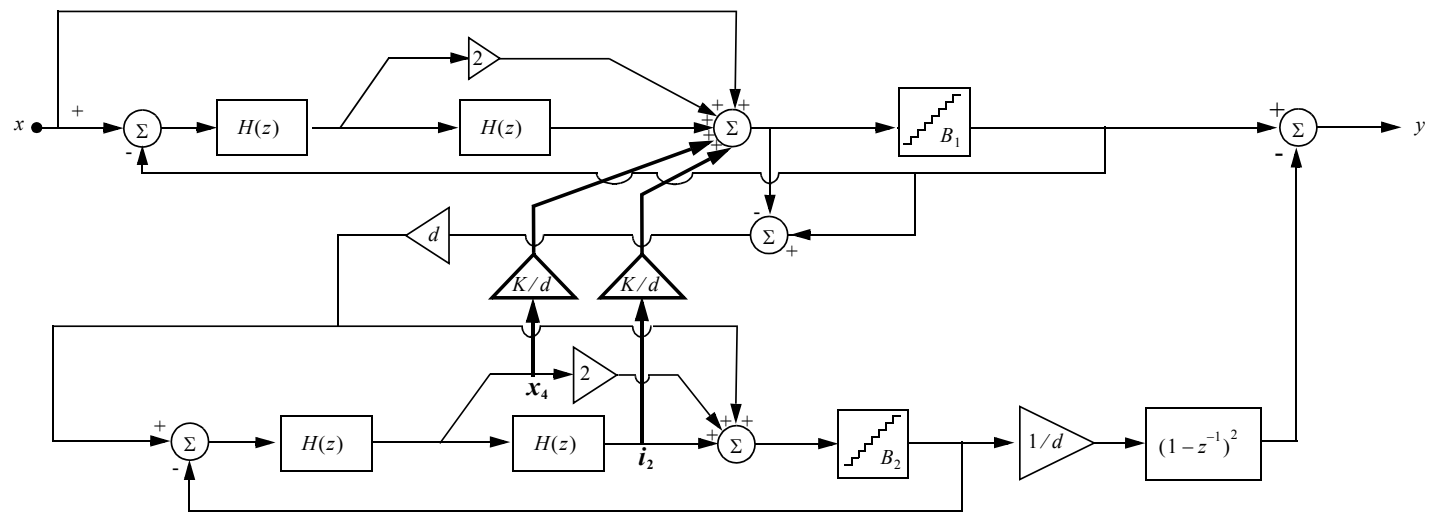

Figure 5. Proposed (II) cascade $\Sigma \Delta \mathrm{M}$ with global resonation. 


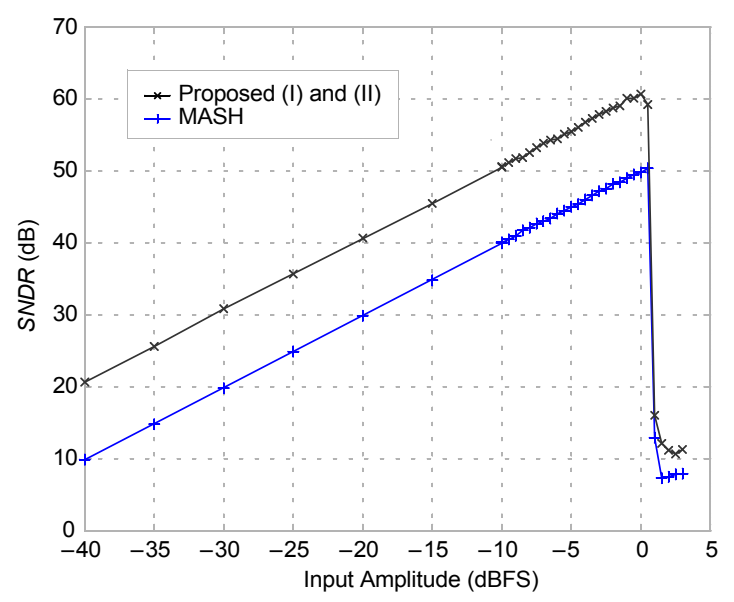

Figure 7. $S N D R$ versus input amplitude.
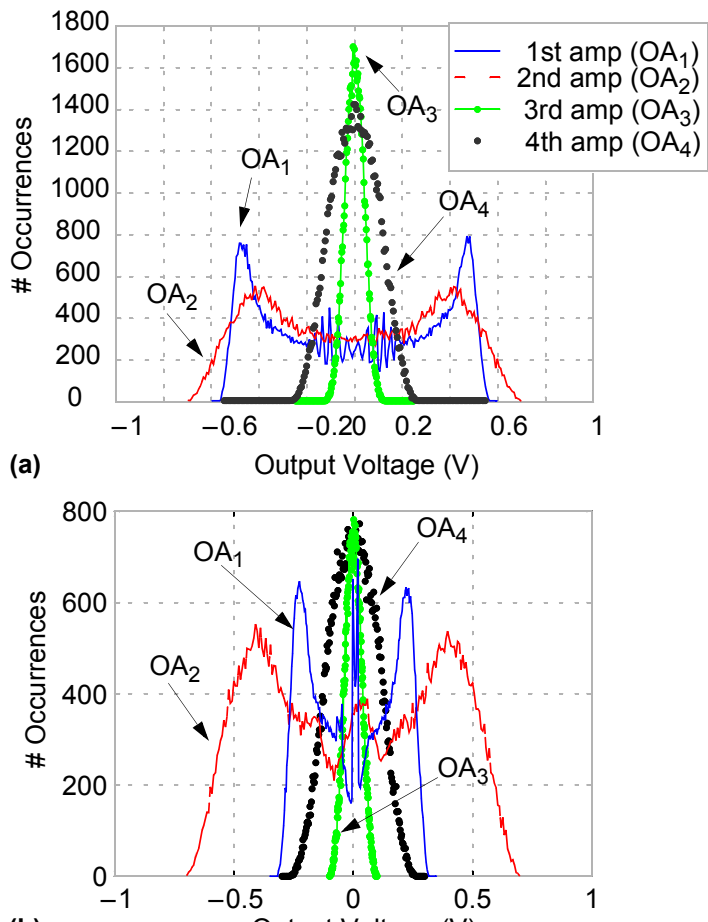

(b)

Output Voltage (V)

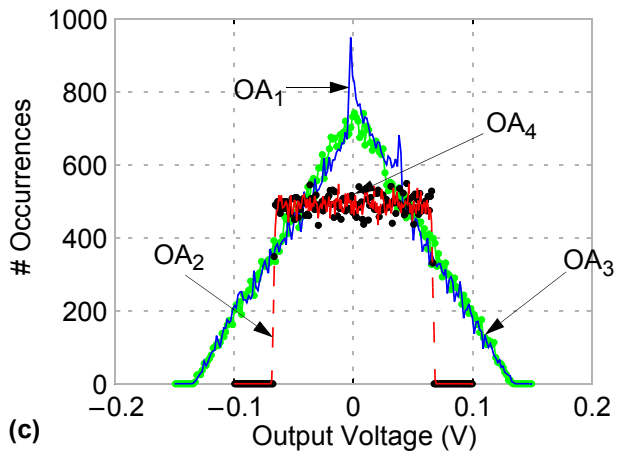

Figure 8. Output swing requirements for the amplifiers of: a) Traditional MASH, b) Global resonation structure in Fig. 2, and c) Proposed (I) and (II) modulators (-6dBFS input level).

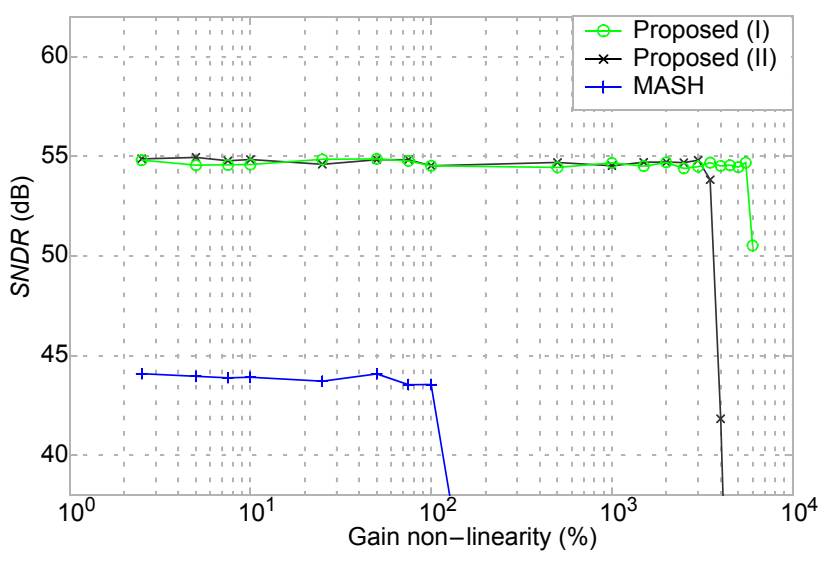

Figure 9. Effect of amplifier non-linearities on the $S D N R$ (-6dBFS input level).

The latter is illustrated in Fig. 9 by plotting the effect of amplifier gain non-linearity on the SNDR. In this simulation, a finite gain of $55 \mathrm{~dB}$ is considered for all the amplifiers, while the gain second-order non-linearity of the first stage amplifiers is varied. Note that, compared with classical cascade architectures, the presented $\Sigma \Delta \mathrm{Ms}$ have much better linearity performance.

\section{CONCLUSIONS}

Two novel topologies of cascade $\Sigma \Delta \mathrm{Ms}$ have been presented. They combine a resonation-based loop filter with a unity STF to achieve very reduced output swing requirements for the amplifiers, whereas increasing the modulator resolution and its robustness with respect to non-linearities as compared to previous approaches. These characteristics make the proposed modulators very appropriate for the implementation of low-voltage wideband $\mathrm{A} / \mathrm{D}$ conversion.

\section{REFERENCES}

[1] R. Schreier: "An empirical study of high-order single-bit delta-sigma modulators". IEEE Trans. on Circuits and Systems-II, issue 8, vol. 40, pp. 461-466, August 1993.

[2] J. Silva et al:: "Wideband low-distortion delta-sigma ADC topology". IEE Electronics Letters, vol. 37, pp. 737-738, June 2001.

[3] J. Silva et al.: "Low-distortion delta-sigma topologies for MASH architectures". Proc. of the 2004 Intl. Symposium on Circuits and Systems (ISCAS), issue 1, vol.1, pp. 1144-1147, May 2004.

[4] J. Markus et al.: "An efficient Delta-Sigma ADC architecture for low oversampling ratios”. IEEE Transactions on Circuits and Systems-I, vol. 51, pp. 63-71, 2004

[5] X. Yuan et al.: "Improved Wideband Low Distortion Cascaded Delta-Sigma Modulator". Proc. of the IEEE Int. Database Engineering \& Application Symposium (IDEAS), 2005.

[6] M. Sanchez-Renedo et al.: "A 2-2 Discrete Time Cascaded $\Sigma \Delta$ Modulator With NTF Zero Using Interstage Feedback". Proc. of the 2006 Intl. Conference on Electronics, Circuits and Systems (ICECS), pp. 954-957, 2006.

[7] A. Gothenberg et al.: "Improved cascaded sigma-delta noise shaper architecture with reduced sensitivity to circuit nonlinearities". IEE Electronics Letters, vol. 38, pp. 683-685, July 2002.

[8] J. Ruíz-Amaya et al.: "High-level synthesis of switched-capacitor, Switched-Current and Continuous-Time $\Sigma \Delta$ Modulators Using SIMULINK-Based Time-Domain Behavioral Models". IEEE Trans. on Circuits and Systems-I, issue 9, vol. 52, pp. 1795-1810, September 2005. 